\title{
Efficient Model for Acoustic Attenuators using the Method of Fundamental Solutions
}

\author{
Edmundo G. de A. Costa \\ Department of Civil Engineering, COPPE - Federal University of Rio de Janeiro, CP 68506, CEP 21945-970, \\ Rio de Janeiro, RJ, Brazil.
}

\section{Luís Godinho}

ISISE, Department of Civil Engineering, University of Coimbra, 3030-788, Coimbra, Portugal.

\author{
José A. F. Santiago and Webe J. Mansur \\ Department of Civil Engineering, COPPE - Federal University of Rio de Janeiro, CP 68506, CEP 21945-970, \\ Rio de Janeiro, RJ, Brazil.
}

\begin{abstract}
(Received 9 September 2015; accepted 5 October 2016)
In this work, the three-dimensional formulation of the Method of Fundamental Solutions (MFS) is applied to model acoustic problems in the frequency domain. This formulation is developed by making use of adequate Green's function defined by the image-source technique, reducing the discretization necessary for the definition of the numerical model. The proposed approach is applied to study the sound attenuation provided by an acoustic attenuating device, consisting of a closed acoustic space located between inlet and outlet tubes. Absorbent properties of the lining materials of the acoustic device, defined using laboratory measurements of their absorption coefficients, are incorporated into the model. The proposed model is verified against reference numerical models based on a boundary integral equation formulation. An experimental validation is also performed, demonstrating that the model adequately simulates the sound propagation under experimental conditions. Numerical applications are then presented to demonstrate the behaviour of the system under different conditions.
\end{abstract}

\section{INTRODUCTION}

The sound field inside closed acoustic spaces is dependent on several factors, such as the excitation frequency of the sound source, its amplitude, its geometry and sound absorption properties. Therefore, several numerical approaches have been developed to deal with this type of problem. Some of these approaches have been applied to the study of attenuating devices that are currently used, for example, in duct systems or in vehicles to reduce the sound emissions and increase user comfort. Indeed, the sound propagation through attenuators is of special practical interest, since these devices are of great importance to reduce the sound that reaches the inhabited spaces within buildings, minimizing the impact of the mechanical noise produced by ventilators and by airflow. Their study has been the subject of research using approaches varying from simplified theoretical formulas based on surface and transfer impedance concepts to much more complex numerical models based on finite elements or boundary elements techniques.

Many analytical and numerical methods were widely used to predict the acoustic attenuation performance of expansion chamber mufflers with the effects of mean flow and higherorder modes (see, for example, the works of Ih and Lee ${ }^{1}$ and Ji et al. ${ }^{2}$ for further details on these effects in duct systems). Expansion chambers were also analyzed by Selamet and $\mathrm{Ji}^{3}$ using both analytical, numerical, and experimental techniques for assessing the importance of extended inlets/outlets in the case of circular expansion chambers. Later, Xu et al. ${ }^{4}$ analyzed these devices in the presence of internal lining using different absorptive materials, making use of numerical techniques and validating the method experimentally. Kim and $\mathrm{Ih}^{5}$ also evaluated the performance of lined plenum chambers with rectangular shape, making use of a Rayleigh-Ritz approach. In both works, it was concluded that the thickness and the type of lining play a fundamental role in the final performance of the chamber.

In what concerns silencers or attenuators, usually inserted into HVAC (abbreviation for Heating, Ventilation, Air Conditioning) networks to enable the reduction of noise that reaches climatized spaces, while allowing the adequate air flow through the piping system, many researchers have developed modelling strategies, using different approaches. Some examples are the publications from $\mathrm{Wu}$ et al. ${ }^{6}$ proposing boundary element formulations for the analysis of packed silencers, from $\mathrm{Lee}^{7}$ which analyzed in detail the performance of hybrid and dissipative mufflers using the Boundary Element Method (BEM), and from Denia et al. ${ }^{8}$ who analyzed the performance of silencers using Finite Element Methods (FEM) and an analytical approach. Many works have also focused on the development of the BEM and its variants for these types of problems, including more intricate acoustic models ${ }^{9-11}$ and proposing highly efficient numerical approaches to tackle large scale problems. ${ }^{12,13}$ Recently, Hua et al. ${ }^{14}$ demonstrated how transmission and insertion loss for multi-inlet mufflers can be determined using impedance matrix and superposition approaches, and Yang et al. ${ }^{15}$ combined the BEM and the point collocation approach to calculate the transmission loss of silencers in the absence of mean flow and temperature gradient.

The development, mostly in the last 20 years, of Meshless Methods has also widened the range of options to simulate sound propagation in that type of configuration. The MFS is one these numerical methods found in the literature. ${ }^{16,17}$ As with the BEM, the MFS is also based on the use of fundamental solutions, which are, themselves, solution of the governing equation of the problem, but not requiring the numerical and 
analytical integrations that need to be performed in boundary integral equation method (hence the singular or hyper-singular integrals are not evaluated). Works such as those by Godinho et al., ${ }^{18}$ Antnio et al. ${ }^{19}$ or Godinho et al. ${ }^{20}$ have shown that, despite its simplicity, the MFS is accurate and constitutes a good tool for simulation of physical systems.

The MFS has attracted great interest of scientists and researchers, due to its simple mathematical formulation that only requires prior knowledge of the fundamental solutions. The linear superposition of these fundamental solutions were employed to approximate the solution of the problem, assuming the virtual sources to be located outside the domain to avoid singularities in the analyzed space. Many researchers have applied the MFS to problems of acoustics, such as Kondapalli et al., ${ }^{21}$ which were among the first to apply the MFS to the Helmholtz equation in the analysis of acoustic scattering in fluids and solids. Fairweather et al. ${ }^{22}$ have also described and analyzed the MFS for the numerical solution to the acoustic scattering problem in fluid and solid media. Alves and Valtchev ${ }^{23}$ compared the plane wave method and MFS for acoustics waves scattering and concluded that the first method can be seen as an asymptotic case of the MFS.

One of the main difficulties in the application of the MFS was the determination of the position of the pseudo-boundary, on which the singularities in terms of which the approximation is expressed, were placed. Therefore, Karageorghis ${ }^{24}$ has proposed a simple practical algorithm for determining an estimate of the pseudo-boundary which yields the most accurate MFS approximation when the method is applied to certain boundary value problems. Later, Godinho et al. ${ }^{20}$ analyzed the behaviour of the MFS numerical frequency domain approach with regards to stability, accuracy, and efficiency, applying strategies for improving stability and accuracy of the method such as the use of different distributions of collocation points and virtual sources or a singular value decomposition (SVD) solver. These strategies allow for the conclusion that the concentration of the collocation points closer to the edges of the model may help in improving this accuracy and that for the analyzed test cases, it is possible to obtain the same level of accuracy using a much smaller equation system with the MFS than with the BEM and that the MFS is a very interesting tool to efficiently predict the sound field inside closed acoustic spaces in the frequency domain.

In the present paper, the authors make use of a numerical approach in the frequency domain, based on the threedimensional formulation of the MFS to calculate the attenuation of the sound field within an acoustic attenuating device. Here, the effect of airflow is not considered. The proposed model considers the symmetry of the problem, modelling thus only a quarter of the numerical model. Moreover, the inner boundary (symmetry planes) of the proposed model require no discretization due to the use of a Green's function which incorporates the appropriate boundary conditions related to symmetry of the problem. The absorbing properties of a possible lining material are incorporated in the numerical model, by means of impedance (Robin) boundary conditions. To validate the numerical implementation of the MFS model, the results are compared with the results obtained in laboratory tests. Finally, a study detailing the behaviour of the acoustic silencer through a set of simulations of their performance considering different absorbing properties of the materials is presented. In this study, the possibility of using lining materials previously characterized by means of laboratory measurements of their absorption coefficients is also discussed.
2. MATHEMATICAL FORMULATION OF THE PROBLEM

\subsection{Sound Propagation in 3D Closed Space}

The propagation of sound within a three-dimensional space can be mathematically represented in the frequency domain by the Helmholtz differential equation:

$$
\nabla^{2} p(\mathbf{x})+k^{2} p(\mathbf{x})=0
$$

where $p(\mathbf{x})$ is acoustic pressure, $k=\omega / c$ is the wave number, with $\omega$ being the angular frequency and $c$ the sound propagation velocity within the acoustic medium.

Assuming a source point placed within this propagation domain, at point $\mathbf{x}_{0}$ with coordinates $\left(x_{0}, y_{0}, z_{0}\right)$, it is possible to establish the fundamental solution for the sound pressure $G$ and particle velocity $H$ at a point $\mathbf{x}$ with coordinates $(x, y, z)$, which can be written respectively as:

$$
\begin{gathered}
G\left(\mathbf{x}, \mathbf{x}_{0}, k\right)=\frac{e^{-1 k r}}{4 \pi r} ; \\
H\left(\mathbf{x}, \mathbf{x}_{0}, k, \mathbf{n}\right)=\frac{1}{\rho \omega} \frac{(-1 k r-1) e^{-1 k r}}{4 \pi r^{2}} \frac{\partial r}{\partial \mathbf{n}}
\end{gathered}
$$

where $r=\sqrt{\left(x-x_{0}\right)^{2}+\left(y-y_{0}\right)^{2}+\left(z-z_{0}\right)^{2}}$, $\mathbf{n}$ represents the direction along which the particle velocity is to be calculated, $\rho$ is the density of the acoustic medium and $i=\sqrt{-1}$.

\subsection{Formulation of the Method of Fundamental Solutions}

Using the Method of Fundamental Solutions, the frequency domain response inside a 3D enclosure is computed as a linear combination of fundamental solutions for a set of virtual sources. These sources are placed outside the domain of interest, to avoid singularities. Thus, at point $\mathrm{x}$ within the propagation domain, the sound pressure field can be written as:

$$
p(\mathbf{x}, k)=\sum_{j=1}^{N V S} A_{j} G\left(\mathbf{x}, \mathbf{x}_{j}, k\right)
$$

where $\mathbf{x}_{j}$ represents the coordinates of the virtual sources $\left(x_{j}, y_{j}, z_{j}\right), A_{j}$ are the unknown amplitudes of the virtual sources which are computed by imposing the appropriate boundary conditions at a set of $N C P$ points (collocation points) placed along the surfaces, $N V S$ is the number of virtual sources and $G\left(\mathbf{x}, \mathbf{x}_{j}, k\right)$ is the fundamental solution at point $\mathbf{x}$ for a virtual source placed at $\mathbf{x}_{j}$. The particle velocity field can be expressed in a similar way. In this work an equal number of collocation points and virtual sources is assumed, which allows obtaining a $N V S \times N V S$ system. This system is built by prescribing at each collocation point $\mathbf{x}$, placed in the boundaries of the closed space, Neumann and Robin conditions. By applying this procedure one obtains,

$$
\sum_{j=1}^{N V S} A_{j} H\left(\mathbf{x}, \mathbf{x}_{j}, k, \mathbf{n}\right)=\overline{v_{n}}(\mathbf{x}, k)
$$

$$
\sum_{j=1}^{N V S} A_{j} G\left(\mathbf{x}, \mathbf{x}_{j}, k\right)=\bar{Z} \sum_{j=1}^{N V S} A_{j} H\left(\mathbf{x}, \mathbf{x}_{j}, k, \mathbf{n}\right)
$$




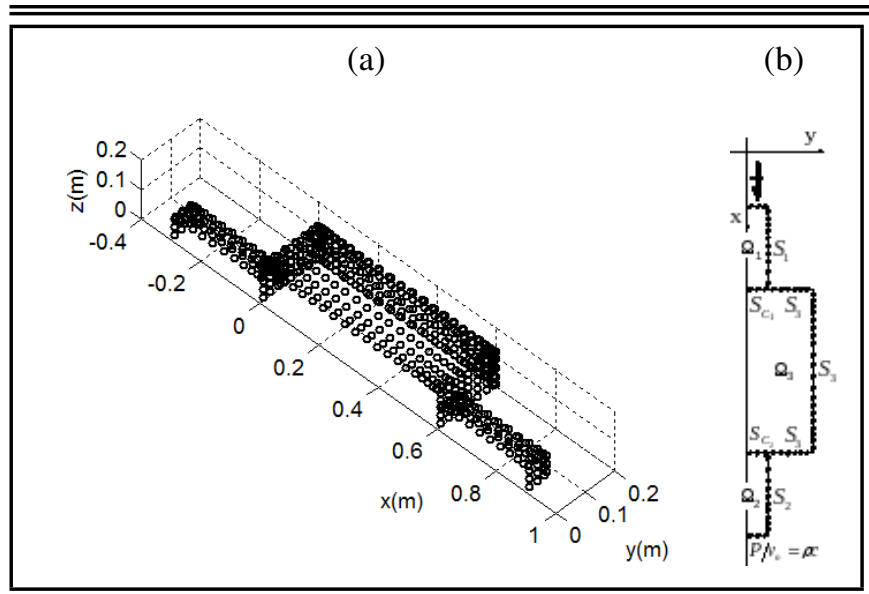

Figure 1. Discretization of a quarter of the model: (a) schematic representation 3-D and (b) schematic representation 2-D.

where $\overline{v_{n}}(\mathbf{x}, k)$ is the normal component of the particle velocity and $\bar{Z}$ is the surface impedance.

If the problem involves more than one sub-domain, then the MFS equation (Eq. (4)) must be written for each sub-domain and continuity conditions must be enforced at interfaces between sub-domains. These continuity conditions can be written as:

$$
\begin{gathered}
p^{+}=p^{-} \quad \text { in } \quad S_{c} \\
v_{n}^{+}=-v_{n}^{-} \quad \text { in } \quad S_{c}
\end{gathered}
$$

where $S_{c}$ is a common interface between the sub-domains, $p$ is the acoustic pressure, and $v_{n}$ is the particle velocity along the normal direction pointing outwards of each sub-domain (i.e. with opposing directions between sub-domains).

In this work, the proposed model has symmetry in relation to the two planes $x y$ and $x z$, as shown in Fig. 1a. Due to this symmetry, it is possible to reduce the discretization to a quarter of the physical model if the following Green's function is assumed, written as:

$$
G\left(\mathbf{x}, \mathbf{x}_{0}, k\right)=\frac{e^{-1 k r_{1}}}{4 \pi r_{1}}+\frac{e^{-1 k r_{2}}}{4 \pi r_{2}}+\frac{e^{-1 k r_{3}}}{4 \pi r_{3}}+\frac{e^{-1 k r_{4}}}{4 \pi r_{4}}
$$

where:

$$
\begin{aligned}
& r_{1}=\sqrt{\left(x-x_{0}\right)^{2}+\left(y-y_{0}\right)^{2}+\left(z-z_{0}\right)^{2}} ; \\
& r_{2}=\sqrt{\left(x-x_{0}\right)^{2}+\left(y-y_{0}\right)^{2}+\left(z+z_{0}\right)^{2}} ; \\
& r_{3}=\sqrt{\left(x-x_{0}\right)^{2}+\left(y+y_{0}\right)^{2}+\left(z-z_{0}\right)^{2}} ; \\
& r_{4}=\sqrt{\left(x-x_{0}\right)^{2}+\left(y+y_{0}\right)^{2}+\left(z+z_{0}\right)^{2}} .
\end{aligned}
$$

To model the proposed problem, we considered that the problem to be solved incorporated three sub-domains: $\Omega_{1}$ and $\Omega_{2}$ corresponding to the entrance (inlet) and exit (outlet) of the silencer; $\Omega_{3}$ corresponding to the inner domain of the silencer. The inlet and outlet surfaces of the silencer are designated as $S_{1}$ and $S_{2}$, respectively, while the inner surface of the silencer is designated as $S_{3}$; the common interface (where continuity of pressure and velocity is imposed) between sub-domains $\Omega_{1}$ and $\Omega_{3}$ is designated as $S_{C_{1}}$ and the common interface between sub-domains $\Omega_{2}$ and $\Omega_{3}$ is designated as $S_{C_{2}}$, as illustrated in Fig. 1b.

In this numerical model, the excitation source is introduced by means of ascribing a boundary condition of $v_{n}=10^{-3} \mathrm{~m} / \mathrm{s}$ along the direction of the inward normal, at the inlet tube; on the opposite face (outlet tube), an anechoic termination is considered by $p / v_{n}=\rho c$.

For this configuration, two variants were considered. The first one considers totally rigid internal surfaces for the silencer, and thus the corresponding boundary conditions can be written as:

$$
\begin{gathered}
v_{n}^{\Omega_{1}}=0 \mathrm{~m} / \mathrm{s} \quad \text { in } \quad S_{1} ; \\
v_{n}^{\Omega_{2}}=0 \mathrm{~m} / \mathrm{s} \quad \text { in } \quad S_{2} ; \\
v_{n}^{\Omega_{3}}=0 \mathrm{~m} / \mathrm{s} \quad \text { in } \quad S_{3} .
\end{gathered}
$$

In the second, the internal surfaces of the silencer are considered to be lined with an absorptive material, and thus the corresponding boundary condition can be written as:

$$
\begin{array}{lll}
p^{\Omega_{1}} / v_{n}^{\Omega_{1}}=\bar{Z} & \text { in } \quad S_{1} ; \\
p^{\Omega_{2}} / v_{n}^{\Omega_{2}}=\bar{Z} & \text { in } \quad S_{2} ; \\
p^{\Omega_{3}} / v_{n}^{\Omega_{3}}=\bar{Z} & \text { in } & S_{3} .
\end{array}
$$

where $\bar{Z}$ is the impedance of the material calculated by an absorption coefficient. This coefficient can be evaluated experimentally, appealing, for example, by impedance tube method. ${ }^{25}$

In both analyses, the equilibrium and continuity condition of the two interfaces between sub-domains of the numerical model can be written as:

$$
\begin{gathered}
p^{\Omega_{1}}=p^{\Omega_{3}} \quad \text { in } \quad S_{C_{1}} ; \\
v_{n}^{\Omega_{1}}=-v_{n}^{\Omega_{3}} \quad \text { in } \quad S_{C_{1}} ; \\
p^{\Omega_{2}}=p^{\Omega_{3}} \quad \text { in } \quad S_{C_{2}} ; \\
v_{n}^{\Omega_{2}}=-v_{n}^{\Omega_{3}} \quad \text { in } \quad S_{C_{2}} .
\end{gathered}
$$

Considering that $N V S_{1}$ virtual sources are distributed around the domain $\Omega_{1}, N V S_{2}$ virtual sources are placed around the domain $\Omega_{2}$, and that $N V S_{3}$ virtual sources are placed around the domain $\Omega_{3}$, the acoustic pressure field inside each domain can be written as:

$$
\begin{array}{ll}
p(\mathbf{x}, k)_{\Omega_{1}}=\sum_{j=1}^{N F V_{1}} A_{j} G\left(\mathbf{x}, \mathbf{x}_{1, j}, k\right) & \text { for } x \text { in } \Omega_{1} ; \\
p(\mathbf{x}, k)_{\Omega_{2}}=\sum_{j=1}^{N F V_{2}} B_{j} G\left(\mathbf{x}, \mathbf{x}_{2, j}, k\right) & \text { for } x \text { in } \Omega_{2} ; \\
p(\mathbf{x}, k)_{\Omega_{3}}=\sum_{j=1}^{N F V_{3}} C_{j} G\left(\mathbf{x}, \mathbf{x}_{3, j}, k\right) & \text { for } x \text { in } \Omega_{3} .
\end{array}
$$

where $A_{j}, B_{j}$, and $C_{j}$ are the amplitudes of the virtual sources placed around the domains $\Omega_{1}, \Omega_{2}$, and $\Omega_{3}$.

Assuming that the number of collocation points exclusively in $S_{1}, S_{2}$ and $S_{3}$ is $N P C_{S 1}, N P C_{S 2}$ and $N P C_{S 3}$, respectively, and that the number of collocation points on the coupling interfaces $S_{C_{1}}$ and $S_{C_{2}}$ is $N P C_{B C_{1}}$ and $N P C_{B C_{2}}$, respectively, after imposing the boundary conditions, a $\left(N P C_{S_{1}}+N P C_{S_{2}}+N P C_{S_{3}}+2 N P C_{B C_{1}}+\right.$ $\left.2 N P C_{B C_{2}}\right) \times\left(N F V_{1}+N F V_{2}+N F V_{3}\right)$ system of equations may be established, allowing the calculation of the $A_{j}$, $B_{j}$, and $C_{j}$ unknowns. 


\section{MODEL VERIFICATION}

Previous works by the authors Godinho et al. ${ }^{20}$ have shown that the accuracy of the response computed using a 3D MFS model depended on the position of the virtual sources used to simulate the pressure field. The proposal for defining this distance as a function of the average spacing between neighbouring collocation points was previously found to be a good strategy; following previous results in Godinho et al., ${ }^{20}$ positioning the virtual sources at a distance that is between 4 and 8 times this spacing was found to be a good compromise between accuracy and stability in 3D configurations. Given this framework, a preliminary study will be performed here, verifying the behaviour of the proposed method against a reference solution based on the boundary integral equation for a geometric configuration of the acoustic silencer. With the purpose of confirming the adequacy of the position of the virtual sources, and to ensure that meaningful results will be obtained, 4 times the average spacing between collocation points will be employed. Thus, it is considered a parallelepiped space with internal dimensions of $0.60 \mathrm{~m} \times 0.40 \mathrm{~m} \times 0.25 \mathrm{~m}$, filled with air, with density of $1.22 \mathrm{~kg} / \mathrm{m}^{3}$, and sound propagation velocity of $340 \mathrm{~m} / \mathrm{s}$; connected to this space, an inlet and an outlet tube are considered. Assume that all the surfaces of this space are totally rigid, with null normal particle velocities, except in the entrance of the device, where the boundary condition is $v=1 \mathrm{~m} / \mathrm{s}$, and in the exit of the device, where the prescribed boundary condition is $v=0 \mathrm{~m} / \mathrm{s}$ (rigid termination).

A schematic representation of the numerical models can be found in Fig. 2. For the MFS model was used a maximum of 554 collocation points, while that for the BEM model a maximum of 880 boundary elements was used.

The proposed MFS model has been used to compute the acoustic pressure inside this system, dividing the propagation medium in three separate domains, and coupling them by imposing full continuity of pressures and particle velocities at the interfaces. In this model, it is assumed a collocation point distribution based on Chebyshev points, closing the extreme points located at the boundary of the surfaces. The basic expression that allows defining these points can be written for the one-dimensional case and, considering npts points to be distributed along a line with unit length, as:

$$
x_{j}=0.5+0.5 \cos \left(\frac{j \pi}{n p t s+1}\right), \quad \text { with } j=1, \ldots, \text { npts } .
$$

In order to illustrate the responses obtained in the verification, Fig. 3 displays the comparison between MFS and BEM results of the sound reduction in the tested configuration. The calculations were performed by assuming frequencies up to $2000 \mathrm{~Hz}$ and a $10 \mathrm{~Hz}$ frequency step. Analysis of the numerical results clearly confirmed that there was an excellent agreement among the two solutions, meaning that the proposed MFS model is accurate in the calculation of the pressure field within the proposed geometry.

In the results shown in Fig. 3, a significantly smaller number of collocation points were employed for the MFS model; this reduction clearly decreased the computational requirements in terms of memory and time while still ensuring good accuracy. Additionally, it must be stressed that no integrations need to be performed, and thus the MFS model can be very efficient from the computational point of view. This behaviour further justified the use of the MFS in these types of problems.

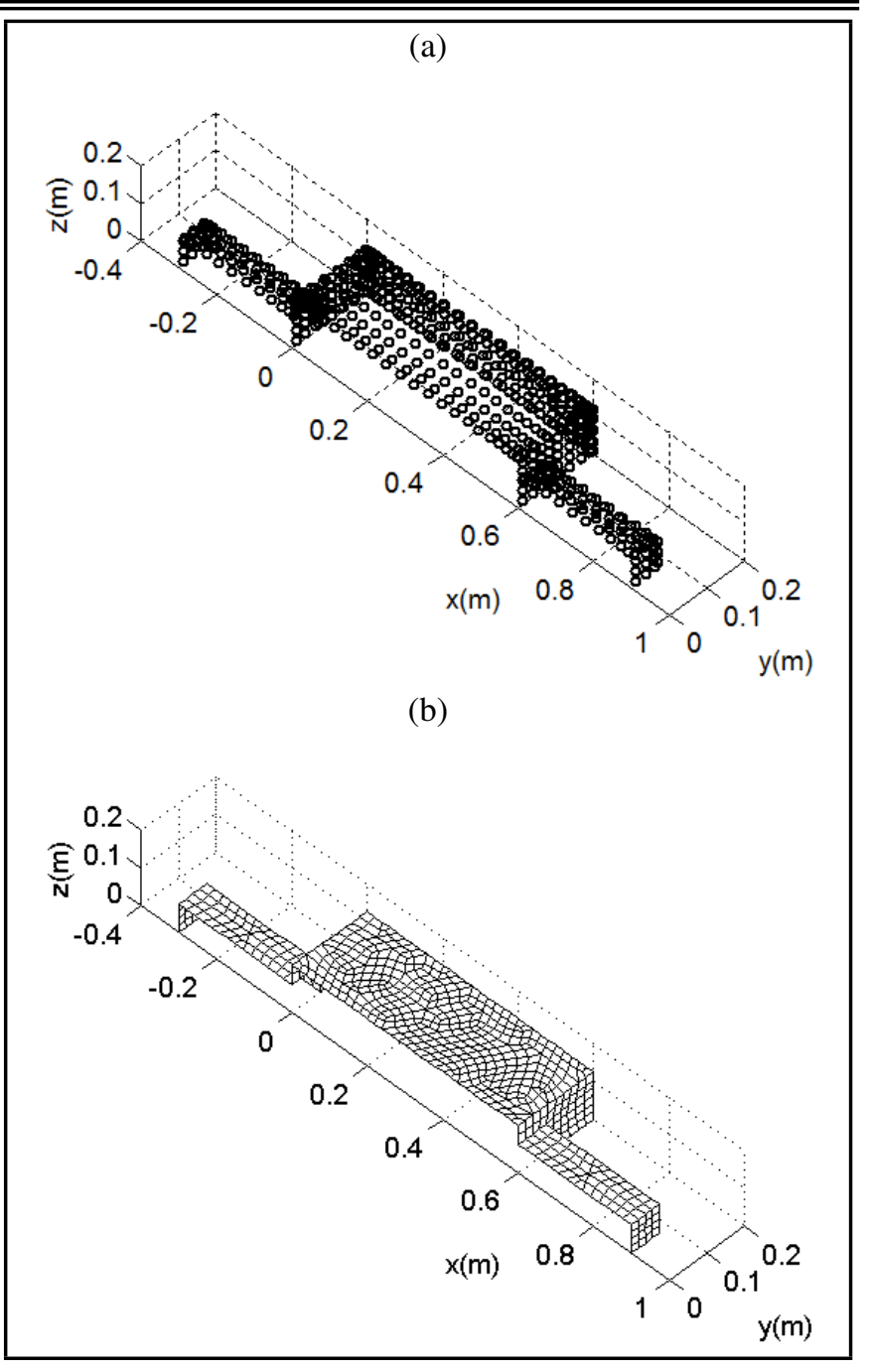

Figure 2. Schematic representation of the numerical models: (a) MFS model and (b) BEM model.

\section{EXPERIMENTAL VALIDATION}

In order to assess the practical applicability of the MFS model in the simulation of sound propagation, it is important to compare the results it provides with those that can be measured experimentally. For this purpose, a simple experimental setup was developed, and subjected to the incidence of sound waves. The sound waves are introduced in this system through an inlet tube with a circular cross-section (with a diameter of $0.05 \mathrm{~m}$ ) centred on one of the faces of the enclosure. The use of an inlet tube with an increased length $(0.90 \mathrm{~m})$ allows for adequate control of the excitation introduced into the system, ensuring that this excitation can be adequately represented as a plane wave. On the face opposite to the source, an exit tube was installed, formed by a short PVC tube $(0.30 \mathrm{~m})$, with a diameter of $0.05 \mathrm{~m}$, with an anechoic termination materialized by a block of mineral wool $0.04 \mathrm{~m}$ thick, with a density of $70 \mathrm{~kg} / \mathrm{m}^{3}$. The sound pressure level was then measured at the entrance (within the impedance tube) and in the exit tubes at opposite faces of the model by means of two microphones.

The signal is acquired using a 2 channel "Symphonie" acquisition system, from $01 \mathrm{~dB}$, connected to two GRAS Sound $\&$ Vibration 40AF microphones. These are free-field microphones subject to an incidence at an angle of $90^{\circ}$, and thus a corrective term should be introduced at each of them, obtained from the characteristic curve provided by the producer (GRAS Sound \& Vibration). However, for this specific microphone 


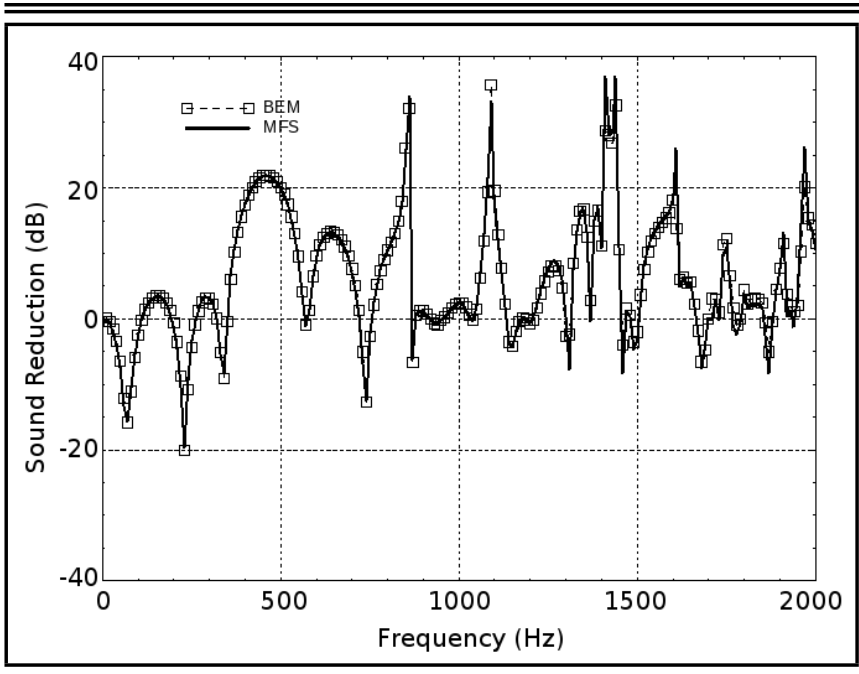

Figure 3. Comparison between MFS and BEM results of the sound reduction in a silencer registered at the entrance and exit tubes.

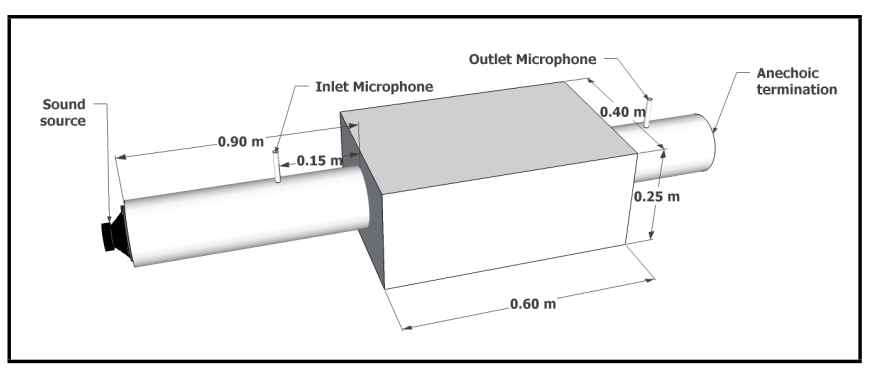

Figure 4. Schematic representation of the laboratorial model used for the experimental validation.

model, the correction factor is approximately null up to $3 \mathrm{kHz}$, and thus it does not influence the measured sound pressure levels within the analyzed frequency range. The response of this system when the impedance tube generates a broad band noise is registered during $6 \mathrm{~s}$, and this test is repeated 5 times. Average SPLs are calculated at each microphone for frequencies up to $2000 \mathrm{~Hz}$. An average SPL reduction is then calculated as the differences between the average SPLs are registered at the entrance and exit tubes. A schematic representation of this model, including its dimensions, can be found in Fig. 4.

This geometry was also modelled to make use of the MFS model considering the symmetry of the problem in relation to the planes $x y$ and $x z$, modelling only $1 / 4$ of the numerical model. In addition, the inner boundary (symmetry planes) of the problem did not need to be discretized, due to use of the Green's function (see Eq. (8)) that considers the boundary conditions of the symmetry of the problem. 600 collocation points are used to model the proposed configuration.

The sound reduction (SR) is calculated by the difference between the registers of the SPLs in the entrance and exit tubes of the acoustic silencer, written as:

$$
\mathrm{SR}=\mathrm{SPL}_{1}-\mathrm{SPL}_{2},(\mathrm{~dB})
$$

where $S P L_{1}$ is the sound pressure level in the entrance of the acoustic silencer and $S P L_{2}$ is the sound pressure level in the exit of the acoustic silencer.

Figure 5 illustrates the comparison between experimental and numerical results computed for the defined configuration. Observing this figure, it is possible to conclude that, in general, there is a good agreement between the measured results and those estimated using the numerical model. In fact, the two

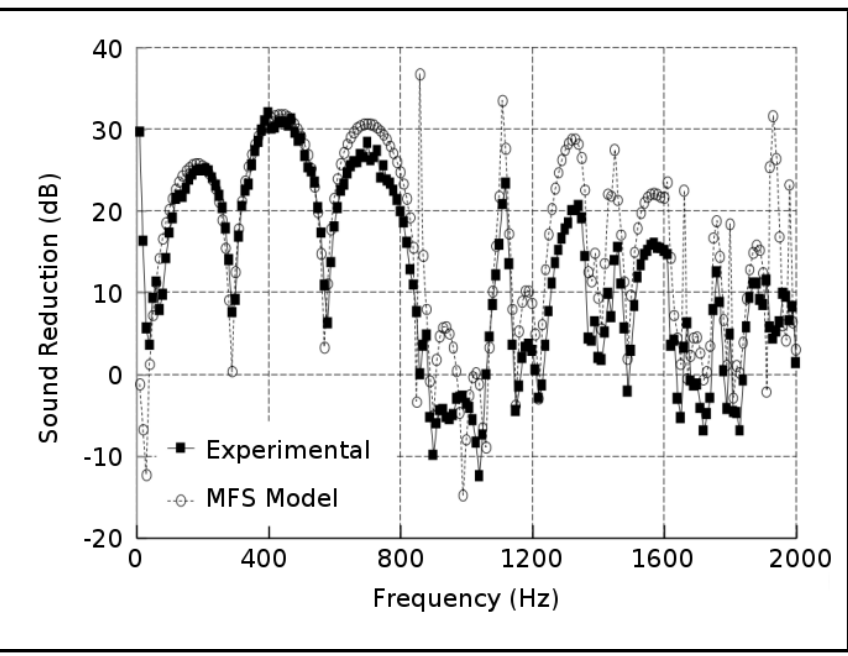

Figure 5. Comparison between experimental and MFS results of the sound reduction in the test device registered at the entrance and exit tubes of $0.05 \mathrm{~m}$.

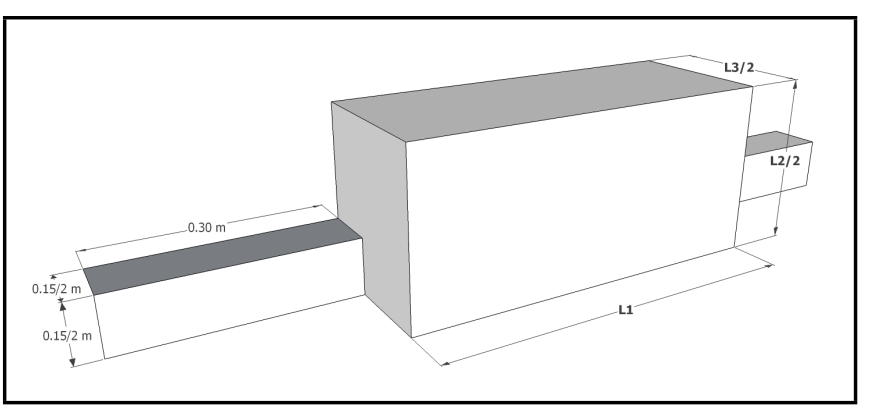

Figure 6. Schematic representation of the analyzed configuration of the sound attenuation device (expansion chamber).

curves have very similar trends, with the position of peaks and valleys matching well between both. Some discrepancies occur in what concerns the involved amplitudes, a situation which can be justified by small differences between the experimental apparatus and the modelled geometry.

\section{NUMERICAL APPLICATIONS}

In order to analyze the mentioned device in greater detail, a set of simulations was performed to analyse its behaviour. In these simulations, the SR was evaluated considering the average of the sound pressures in several receivers localized in the entrance and exit of the acoustic silencer, written as:

$$
\begin{aligned}
\mathrm{SR}=20 \log & \left(\frac{\sum_{i=1}^{n \mathrm{rec}_{1}}\left|\left(p_{1}\right)_{i}\right| / n \mathrm{rec}_{1}}{2.10^{-5} \mathrm{~Pa}}\right) \\
& -20 \log \left(\frac{\sum_{i=1}^{n \mathrm{rec}_{2}}\left|\left(p_{2}\right)_{i}\right| / n \mathrm{rec}_{2}}{2.10^{-5} \mathrm{~Pa}}\right)(\mathrm{dB})
\end{aligned}
$$

where $p_{1}$ is the sound pressure in the entrance, $p_{2}$ is the sound pressure in the exit of the silencer; $n$ rec $_{1}$ and $n \mathrm{rec}_{2}$ are the number of receivers localized in the entrance and exit of the device, respectively. In this analysis, two sets of 16 receivers were considered in the entrance and in the exit of the silencer, equally spaced of $0.01 \mathrm{~m}$ along the $x$-direction (see Fig. 6).

Two different boundary conditions of the acoustic domain of the silencer were considered: the first corresponding to Neumann condition, which consists in considering null velocities in all internal walls of the device; and the second corresponding to Robin conditions able to simulate an absorption coeffi- 


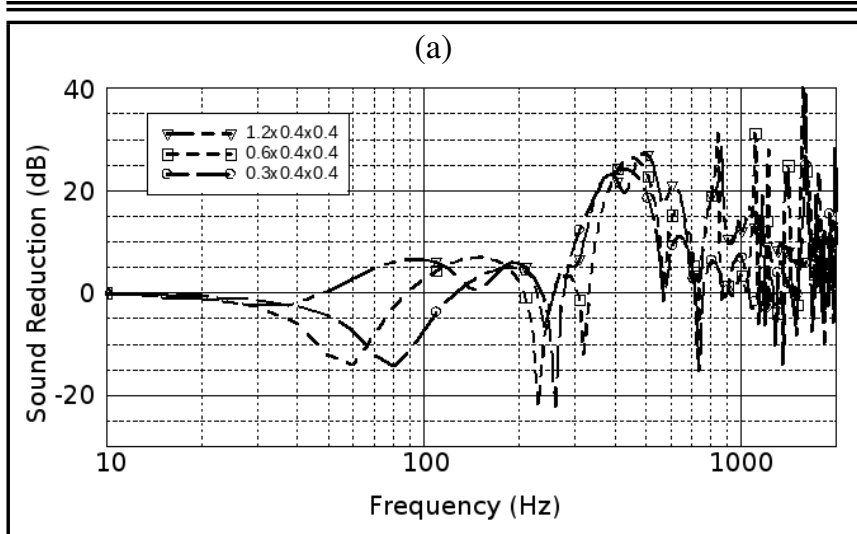

(b)

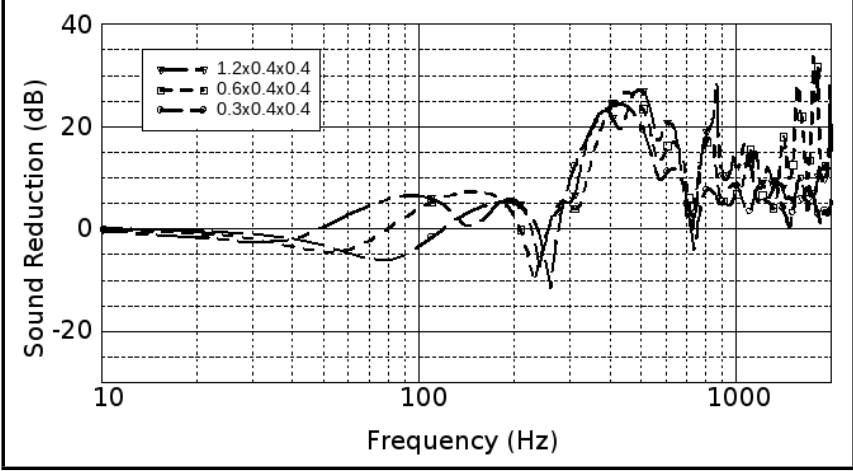

Figure 7. Numerical curves of the sound reduction for a device with a cross section of $0.4 \mathrm{~m} \times 0.4 \mathrm{~m}$, with different lengths: (a) device with rigid walls; (b) device with absorbing walls $(\bar{\alpha}=0.2)$.

cient $\bar{a}$ given as:

$$
\bar{Z}=\rho c \frac{1+\sqrt{1-\bar{\alpha}}}{1-\sqrt{1-\bar{\alpha}}} .
$$

A generic model is here considered, as described in Fig. 6, consisting of a parallelepiped silencer device (an expansion chamber) connected to an inlet and an outlet tube with crosssection of $0.15 \mathrm{~m} \times 0.15 \mathrm{~m}$. The criterion described in the previous sections to define the collocation point distribution in the numerical model, was also used in this chapter.

Figure 7 illustrates the results of the SR (in $\mathrm{dB}$ ) as a function of the frequency, in the range $[10 ; 2000 \mathrm{~Hz}]$ with a frequency step of $10 \mathrm{~Hz}$, for different lengths of the expansion chamber device when its cross-section is $0.4 \mathrm{~m} \times 0.4 \mathrm{~m}$. Two types of model were analyzed, namely with totally reflective internal walls (Fig. 7a) and with its walls lined with an absorbing material with $\bar{\alpha}=0.2$ (Fig. 7b). For both cases, the sound reduction curves exhibit an oscillatory behaviour, associated with the multiple internal reflections that occur within the acoustic space. More oscillations are visible when the larger values of $L_{1}$ are adopted (namely $1.2 \mathrm{~m}$ ), given the lower frequency of internal resonance that occurs in that case. At higher frequencies, a sequence of peaks and dips occurs, and originates a very complex behaviour, alternating attenuation with amplification phenomena. When the internal surfaces are lined, a smoother trend is observed in the sound reduction and those peaks and dips are greatly attenuated. For both cases, and for all values of L1, it can be seen that the attenuation peaks around 500 $\mathrm{Hz}$, with similar values for all configurations. This behaviour seems to be more associated with the cross-section of the device than with its length. Indeed, this can be explained by the fact that the attenuating effect of these devices are based on the contrast between impedances of the inlet/device/outlet.

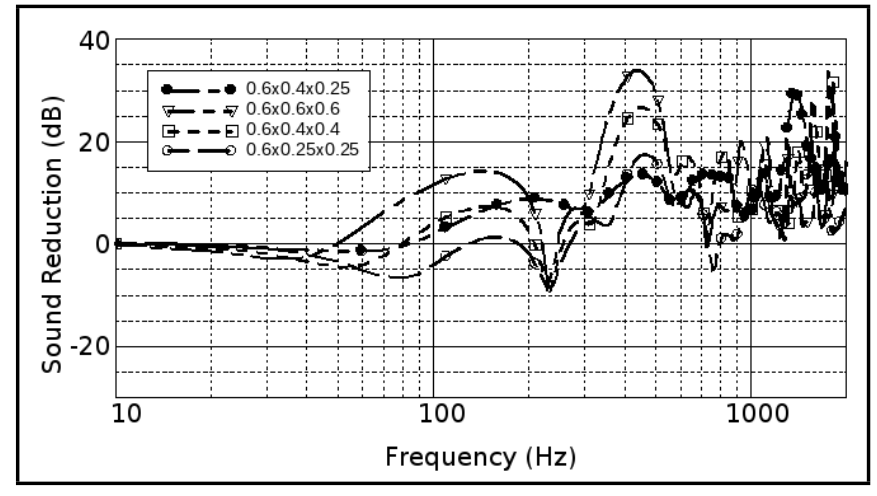

Figure 8. Numerical curves of the sound reduction for varying cross-section dimensions, with $L_{1}=0.6 \mathrm{~m}$ for a device with absorbing walls $(\bar{\alpha}=0.2)$.

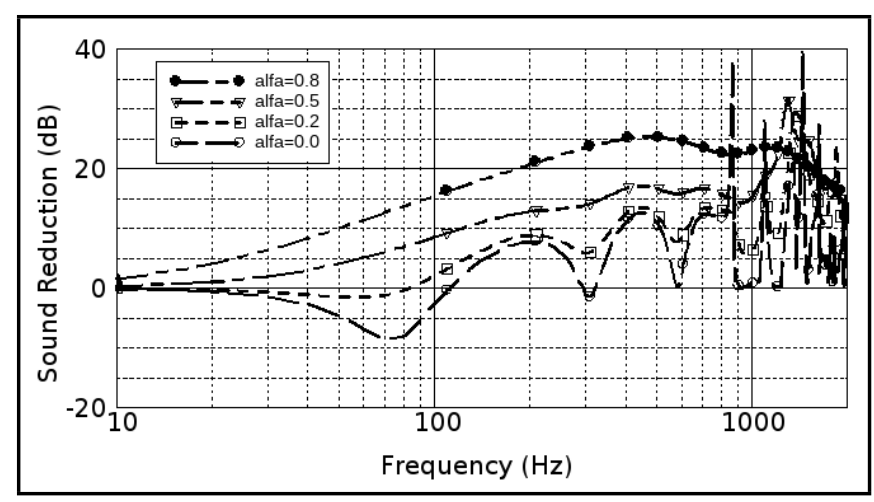

Figure 9. Numerical curves obtained in a device with dimensions $0.6 \mathrm{~m} \times$ $0.4 \mathrm{~m} \times 0.25 \mathrm{~m}$, for the sound reduction for different value of the absorption coefficient $(\bar{\alpha})$.

In Figure 8, the influence of the cross-section of the attenuating device is analyzed, considering different cross-section dimensions $(\mathrm{L} 2 \times \mathrm{L} 3)$, and $\mathrm{L} 1=0.6 \mathrm{~m}$, considering the internal walls with $\bar{\alpha}=0.2$. From the presented curves, it becomes clear that as larger contrasts between the inlet/outlet tubes and the device cross-section are considered, higher sound reduction values may be obtained. Indeed, this confirms the previous explained behaviour, also observed in Fig. 7. It is interesting to note that a somewhat smoother curve is obtained when the cross-section is not square. In fact, a non-square cross-section originates different resonance frequencies along the three orthogonal directions and avoids the strong dips that were otherwise observed.

Figure 9 illustrates the results of the SR for different values of the absorption coefficient, when the attenuation device has dimension $0.6 \mathrm{~m} \times 0.4 \mathrm{~m} \times 0.25 \mathrm{~m}$. In this figure, it is interesting to note that significant differences are registered between the curves of the sound reduction level for the different absorption coefficients. Indeed, observing this figure, smoother curves are progressively obtained for higher values of the absorption coefficient, with the effect of the peaks and dips being greatly attenuated when significant absorption is introduced. For larger sound absorption coefficients, fewer modes propagate inside the silencer and consequently those pronounced peaks and valleys which appear when the internal walls of the silencer are considered to be totally rigid will become increasingly less pronounced.

A more realistic situation was also modelled, considering the sound absorption coefficient as a function of the frequency, following the pattern determined in the laboratory for mineral wool. As usual for porous and fibrous materials, the evaluated sound absorption coefficient varies with the frequency and 


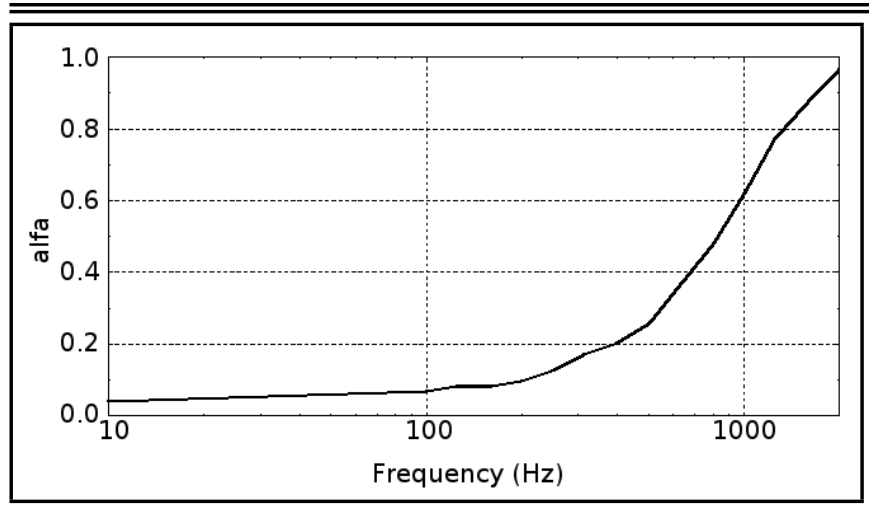

Figure 10. Absorption coefficient obtained for the mineral wool in impedance tube tests, at the Department of Civil Engineering of the University of Coimbra.

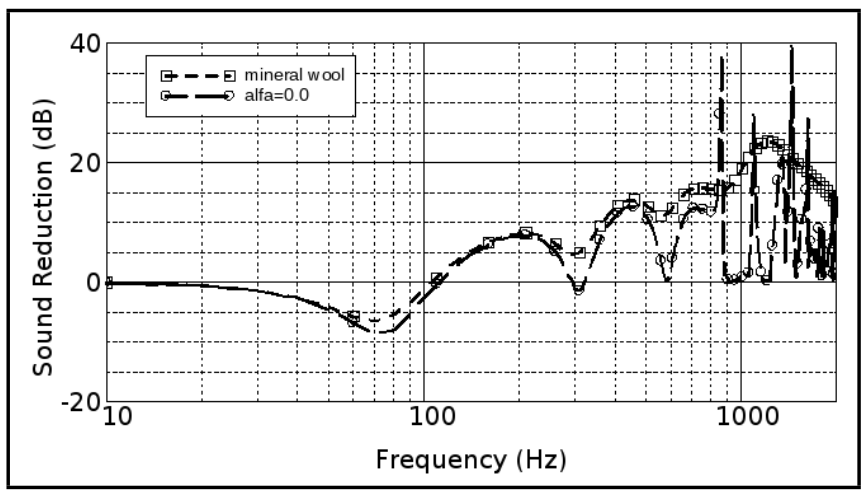

Figure 11. Numerical curves obtained for the sound reduction in an acoustic silencer with entrance and exit tubes with section of $0.15 \mathrm{~m}$, considering the rigid walls of the silencer (null absorption coefficient) and walls of the silencer with lining of mineral wool (absorption coefficient as a function of the frequency).

higher values present only in high frequencies, as can be seen in Fig. 10. The calculated results for this situation can be seen in Fig. 11. The behaviour of the system can now be seen as a mixture of those previously observed in Fig. 9. Indeed, for lower frequencies, the internal walls have nearly no absorption, and the registered sound reduction thus approaches very much that of a rigid device. As frequency increases, the lining material exhibits higher absorption coefficients and thus, the peaks and valleys that occur in the SR curve are progressively less pronounced. Indeed, this type of material tends to smooth the SR curve predominantly at higher frequencies in which the material is more effectively absorbing sound.

To better observe this phenomenon, additional calculations were performed for receivers located over a grid in the plane $x y$ at $z=0.0 \mathrm{~m}$. The respective sound levels are shown in Fig. 12, in which the left column corresponds to the situation in which the silencer has completely rigid inner walls and the right column corresponds to the situation in which the inner walls of the silencer are lined with mineral wool. The results are illustrated for the excitation frequencies of $100 \mathrm{~Hz}$, $500 \mathrm{~Hz}, 1500 \mathrm{~Hz}$, and $2000 \mathrm{~Hz}$. For the case in which the silencer has no absorbent material (internal walls of the silencer are completely rigid), sound levels recorded on the receivers reach higher amplitudes for the higher frequencies of $1500 \mathrm{~Hz}$ and $2000 \mathrm{~Hz}$ (see Figs. 12c1 and 12d1, respectively) due to the reflections that occur within the acoustic silencer. For that situation, the effect of those multiple reflections is clearly visible in Figs. $12 \mathrm{~b} 1$ to $12 \mathrm{~d} 1$, exhibiting the typical modal behaviour of a closed acoustic space. When the internal walls of the silencer are lined with mineral wool, some changes become more apparent in the results. For lower frequencies, a small reduction of the sound levels can be observed at specific locations (see Figs. 12a2 and 12b2), while, for the higher frequencies of $1500 \mathrm{~Hz}$ and $2000 \mathrm{~Hz}$, the attenuation is significantly more pronounced within the attenuating device with reductions around 15 to $30 \mathrm{~dB}$ (see Figs. 12c2 and 12d2) within this space. Since the mineral wool exhibits higher absorption at higher frequencies, lining the inside of the device is very effective at those higher frequencies.

\section{CONCLUSIONS}

A numerical frequency domain approach, based on the three-dimensional formulation of the MFS was used to calculate the attenuation of the sound field within of an acoustic attenuator device in the absence of mean flow. Due to the symmetry of the acoustic problem, only a quarter of the model was discretized. Moreover, the discretization of the inner boundary (symmetry planes) of the model is not required due to the use of the Green's functions that incorporate the boundary conditions related to symmetry of the problem. The numerical model also accounts for possible absorbing properties of the material lining the interior of the device, eventually defined by laboratory measurement of its sound absorption coefficients. The numerical results were validated with the results obtained in the laboratory test. In what concerns the proposed model, it was concluded that it can be quite an interesting tool for providing accurate results with increased computational efficiency when compared to traditional models such as those based on the boundary element model.

The parametric study conducted by evaluating the effect of the dimensions of the device and of the material lining its interior in the sound reduction it provides, revealed interesting behaviours. As expected, it was observed that both increasing the dimensions (in terms of cross-section) and the absorption of the lining material further increases the attenuating capabilities of the silencer device.

\section{ACKNOWLEDGMENTS}

The authors would like to thank CNPq (National Council for Scientific and Technological Development) and FAPERJ (Research Support Foundation of the State of Rio de Janeiro) for providing financial support to this research. This work was also financed by FEDER funds through the Competitivity Factors Operational Programme - COMPETE and by national funds through FCT within the scope of the project POCI-010145-FEDER-007633.

\section{REFERENCES}

1 Ih, J. G. and Lee, B, H. Analysis of Higher Order Mode Effects in the Circular Expansion Chamber with mean flow, Journal of the Acoustical Society of America, 77, 13771388, (1985). https://dx.doi.org/10.1121/1.392029

2 Ji, Z., Ma. Q. and Zhang, Z. Application of the Boundary Element Method to Predicting Acoustic Performance of Expansion Chamber Mufflers with mean Flow, Journal of Sound and Vibration, 173 (1), 57-71, (1994). https://dx.doi.org/10.1006/jsvi.1994.1217 


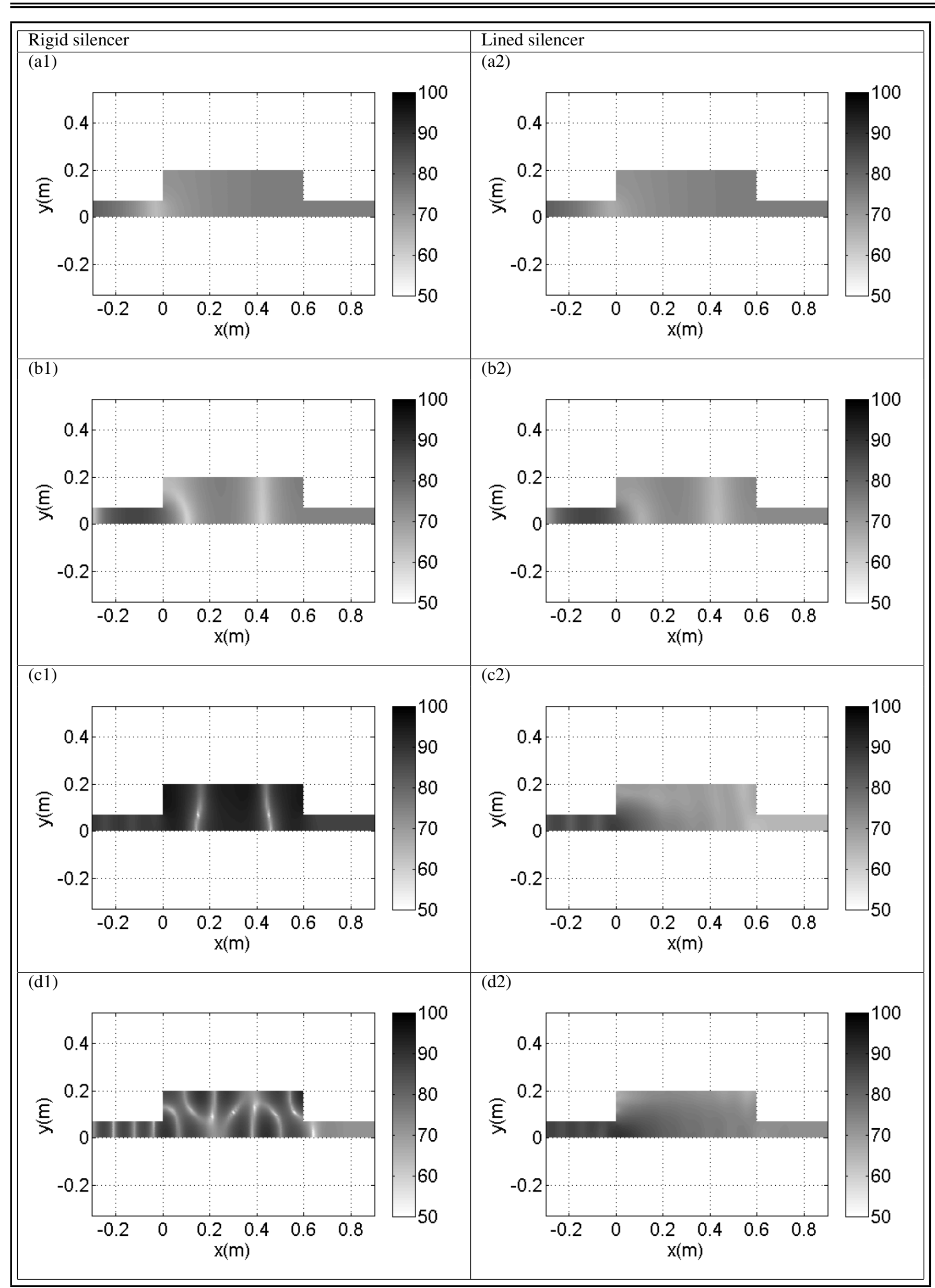

Figure 12. SPL obtained over a grid of receivers localized on the plane xy for the excitation frequencies of: (a) $100 \mathrm{~Hz},(\mathrm{~b}) 500 \mathrm{~Hz}$, (c) $1500 \mathrm{~Hz}$, and (d) $2000 \mathrm{~Hz}$. 
3 Selamet, A., and Ji, Z. L. Acoustic Attenuation Performance of Circular Expansion Chambers with Extended Inlet/Outlet, Journal of Sound and Vibration, 223 (2), 197212, (1999). https://dx.doi.org/10.1006/jsvi.1998.2138

$4 \mathrm{Xu}$, M. B., Selamet, A., Lee, I.-J., and Huff, N. T. Sound Attenuation in Dissipative Expansion Chambers, Journal of Sound and Vibration, 272, 1125-1133, (2004). https://dx.doi.org/10.1016/j.jsv.2003.07.025

5 Kim, H.-J., and Ih, J.-G. Rayleigh-Ritz Approach for Predicting the Acoustic Performance of Lined Rectangular Plenum Chambers, Journal of the Acoustical Society of America, 120 (4), 1859-1870, (2006). https://dx.doi.org/10.1121/1.2336748

6 Wu, T. W., Cheng, C. Y. R., and Zhang, P. A Direct Mixed-Body Boundary Element Method for Packed Silencers, Journal of the Acoustical Society of America, 111, 2566-2572, (2002). https://dx.doi.org/10.1121/1.1476920

7 Lee, I. Acoustic Characteristics of Perforated Dissipative and Hybrid Silencers, PhD Thesis, The Ohio State University, (2005).

8 Denia, F. D., Selamet, A., Fuenmayor, F. J., and Kirby, R. Acoustic Attenuation Performance of Perforated Dissipative Mufflers with Empty Inlet/Outlet Extensions, Journal of Sound and Vibration, 302, 1000-1017, (2007). https://dx.doi.org/10.1016/j.jsv.2007.01.005

9 Ji, Z. L. Boundary Element Acoustic Analysis of Hybrid Expansion Chamber Silencers with Perforated Facing, Engineering Analysis with Boundary Elements, 34 (7), 690-696, (2010). https://dx.doi.org/10.1016/j.enganabound.2010.02.006

10 Jiang, C., Wu, T. W., and Cheng, C. Y. R. A Single-domain Boundary Element Method for Packed Silencers with Multiple Bulk-reacting Sound Absorbing Materials, Engineering Analysis with Boundary Elements, 34 (11), 971-976, (2010). https://dx.doi.org/10.1016/j.enganabound.2010.06.009

11 Chen, Z.-S., Kreuzer, W., Waubke, H., and Svobodnik, A. J. A Burton-Miller Formulation of the Boundary Element Method for Baffle Problems in Acoustics and the BEM/FEM Coupling, Engineering Analysis with Boundary Elements, 35 (3), 279-288, (2011). https://dx.doi.org/10.1016/j.enganabound.2010.11.004

12 Li, S., and Huang, Q. A Fast Multipole Boundary Element Method based on the Improved Burton-Miller Formulation for Three-dimensional Acoustic Problems, Engineering Analysis with Boundary Elements, 35 (5), 719-728, (2011). https://dx.doi.org/10.1016/j.enganabound.2010.12.004

13 Wang, X., Zhang, J., Zhou, F., and Zheng, X. An Adaptive Fast Multipole Boundary Face Method with Higher Order Elements for Acoustic Problems in Three-dimension, Engineering Analysis with Boundary Elements, 37 (1), 144-152, (2013). https://dx.doi.org/10.1016/j.enganabound.2012.09.006

14 Hua, X., Jiang, C., Herrin, Wu, T. W. Determination of Transmission and Insertion Loss for
Multi-inlet Mufflers using Impedance Matrix and Superposition Approaches with Comparisons, Journal of Sound and Vibration, 333, 5680-5692, (2014). https://dx.doi.org/10.1016/j.jsv.2014.06.016

15 Yang, L., Ji, Z. L., Wu, T. W. Transmission Loss Prediction of Silencers by using Combined Boundary Element Method and Point Collocation Approach, Engineering Analysis with Boundary Elements, 61, 265-273, (2015). https://dx.doi.org/10.1016/j.enganabound.2015.08.004

16 Golberg, M. A., and Chen, C. S. The Method of Fundamental Solutions for Potential, Helmholtz and Diffusion Problems, in Golberg, M. A., (Ed.), Boundary Integral Methods: Numerical and Mathematical Aspects, WIT Press \& Computational Mechanics Publications, 103-176, (1999).

17 Cho, H., Golberg, M., and Muleshkov, A., and Li, X. Trefftz Methods for Time Dependent Partial Differential Equations, CMC: Computers, Materials and Continua, 1 (1), 1-38, (2004).

18 Godinho, L., Branco, F. G., and Amado Mendes, P. Simulation of Sound Propagation between Two Closed Spaces Using the Method of Fundamental Solutions, in Topping, B. H. V., and Papadrakakis, M. (Eds.), Proceedings of the Ninth International Conference on Computational Structures Technology (Paper 81), Civil-Comp Press, Stirlingshire, Scot-land, (2008).

19 Antnio, J., Tadeu, A., and Godinho, L. A Threedimensional Acoustics Model using the Method of Fundamental Solutions, Engineering Analysis with Boundary Elements, 32, 525-531, (2008). https://dx.doi.org/10.1016/j.enganabound.2007.10.008

20 Godinho, L. M. C., Costa, E. G. A., Pereira, A. S. C., and Santiago, J. A. F. Some Observations on the Behavior of the method of fundamental solutions in 3D Acoustic Problems, International Journal of Computational Methods, 9 (4), 1250049, (2012). https://dx.doi.org/10.1142/S0219876212500491

21 Kondapalli, P. S., Shippy, D. J., and Fairweather, G. Analysis of Acoustic Scattering in Fluids and Solids by the Method of Fundamental Solutions, Journal of the Acoustical Society of America, 91 (4), 1844-1854, (1992). https://dx.doi.org/10.1121/1.403714

22 Fairweather, G., and Karageorghis, A. The Method of Fundamental Solutions for Elliptic Boundary Value Problems, Advances in Computational Mathematics, 9, 69-95, (1998). https://dx.doi.org/10.1023/A:1018981221740

23 Alves, C. J. S., and Valtchev, S. S. Numerical Comparison of Two Meshfree Methods for Acoustic Wave Scattering, Engineering Analysis with Boundary Elements, 29, 371-382, (2005). https://dx.doi.org/10.1016/j.enganabound.2004.09.008

24 Karageorghis, A. A Practical Algorithm for Determining the Optimal Pseudo-Boundary in the Method of Fundamental Solutions, Advances in Applied Mathematics and Mechanics, 1 (4), 510-528, (2009).

25 Silva, J., Godinho, L., Pereira, A. Determinação Experimental da Absorção Sonora de Materiais utilizando Amostras de Dimensões Reduzidas, Acústica 2008, Universidade de Coimbra, Coimbra, Portugal, (2008). 